\title{
In-situ-by-Ex-situ: FIB-less Preparation of Bulk Samples on Heating Membranes for Atomic Resolution STEM Imaging
}

\author{
Weizong Xu, Everett D. Grimley and James M. LeBeau
}

Department of Materials Science and Engineering, North Carolina State University, Raleigh, NC 27695, USA

Recent advances in in-situ electron microscopy have enabled materials characterization to a new level. Namely, advanced in-situ electron microscopy sample holders integrated with heating, liquid, or gas environmental cells have permitted the investigation of kinetic processes while maintaining atomic resolution [1]. Even with these impressive advancements, however, much in-situ work has been performed on nanoparticles and their supporters by dispersing liquid drops onto grids. For these samples, it is often difficult to precisely align along a low order zone axis, which is required for atomic resolution STEM imaging. For bulk samples prepared by FIB, the Ga contamination and/or the protective layer can cause unwanted reactions that prevent observation of pristine surfaces and interfaces. As a result, few works have successfully shown atomic resolution STEM imaging at temperatures higher than $700{ }^{\circ} \mathrm{C}$ for membrane based devices [1, 2]. Given these complications, quantitative, or even qualitative, structural and chemical analysis of bulk samples at high temperature remains challenging.

In this presentation, we highlight an ex-situ specimen preparation method for in-situ membrane based heating devices. An overview of the process is shown schematically in Figure 1 for specimen mounting onto a Protochips Inc. heating membrane. First, the material is wedge-polished and then ion-milled to final thinness. The sample is then fractured into pieces, which are typically in the range of $20-100 \mu \mathrm{m}$ in size. A glass needle is then used to select thin regions from amongst the debris, similar to ex-situ FIB lift-out approach utilizing static and Van de Walls forces [3]. Compared with FIB lift-out sample preparation, the methodology enables the observation of large areas of bulk samples with minimum cost and avoids $\mathrm{Ga}^{+}$damage/contamination for near pristine surface imaging. Furthermore, sample fixing on membrane using $\mathrm{Pt} / \mathrm{W}$ deposition is not necessary because of the large sample size. This further eliminates $\mathrm{Pt} / \mathrm{W}$ contamination during the FIB/e-beam deposition.

Pairing the above approach with a Protochips Aduro double tilt holder, we readily demonstrate atomic resolution STEM imaging at "ultra" high temperatures close to the limit of membrane-based chip (Protochips Inc.) of $1200{ }^{\circ} \mathrm{C}$. In Figure 2, we show that the sample preparation method can be successfully applied to a wide range of materials including $\mathrm{Si}, \mathrm{SrTiO}_{3}, \mathrm{MgO}$ and YSZ. It is important to note that the images are as acquired, without any post-acquisition adjustments, even though some jittering of the signal is observed. In fact, we found jittering to be a limiting factor for atomic resolution, which degraded from the room temperature resolution of about $0.8 \AA$ to $1.2-1.4 \AA$ at these temperatures.

Based on these results, we show that this methodology can extend in-situ STEM imaging to reveal atomically resolved structural and chemical information during heating to high temperatures. An example can be seen in Figure 3(a) and (b) for the polar $\mathrm{MgO}$ (111) surface where the atomic configurations of $\mathrm{Mg}$ and $\mathrm{O}$ atoms are revealed. Interestingly, a unique stacking fault like surface reconstruction is directly revealed with oxygen termination, which to our knowledge has not been observed previously. Perhaps most striking, the detailed nature of the atomic configuration is generally 
not accessible by other surface characterization methods such as scanning tunneling microscopy, due to the insulating nature of $\mathrm{MgO}$. Finally, the methodology is widely applicable to other membrane-based in-situ devices, enabling not only heating experiments but also environmental, electrical biasing, etc.

\section{References:}

[1] L. F. Allard, et al, Microscopy research and technique, 72(2009), p. 208.

[2] M. Chi, et al, Nature Communications, 6(2015), p. 8925.

[3] L. A. Giannuzzi, et al, Microscopy and Microanalysis, 21(2015), p. 1034.

[4] This work is supported by the National Science Foundation (Grant: DMR-1350273). The authors also acknowledge the Analytical Instrumentation Facility (AIF) at North Carolina State University, which is supported by the State of North Carolina and the National Science Foundation.

a

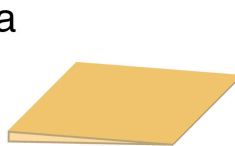

b

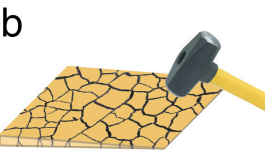

C

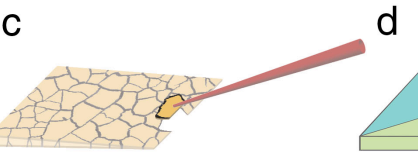

e

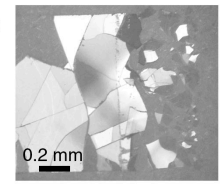

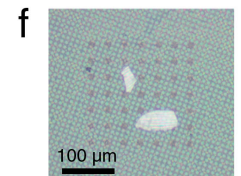

Figure 1. (a) Wedge polished sample is (b) broken into pieces, (c) where thin areas are lifted out and (d) attached to the Protochips heating membrane. (e, f) Experimental illustrations of (c) and (d), respectively.

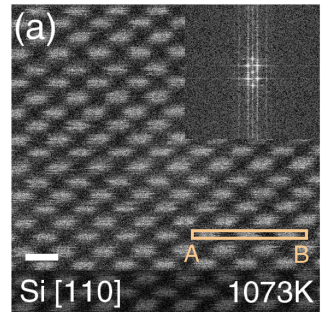

(e)

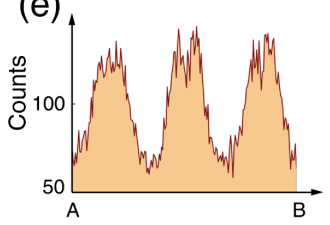

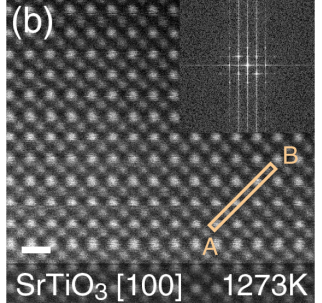

(f)

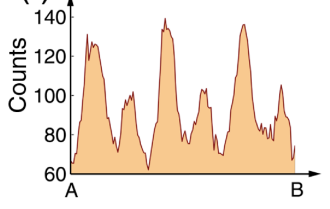

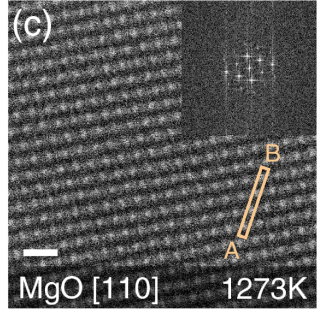

(g)

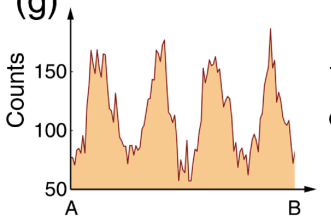

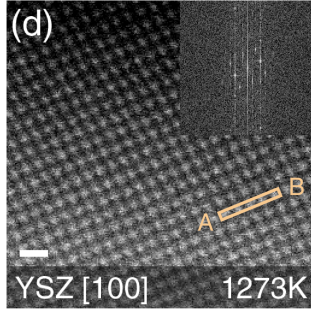

(h)

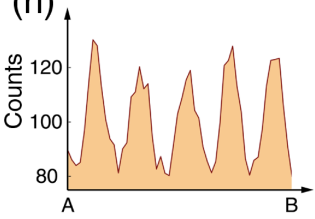

Figure 2. High temperature atomic STEM observation of typical substrate materials (a) Si @800 ${ }^{\circ} \mathrm{C}$ (b) STO@1000 ${ }^{\circ} \mathrm{C}$, (c) $\mathrm{MgO} @ 1000{ }^{\circ} \mathrm{C}$ (d) YSZ @ $1000^{\circ} \mathrm{C}$ and their corresponding intensity profiles (e-h) along path from A to B, scale bar $0.5 \mathrm{~nm}$.
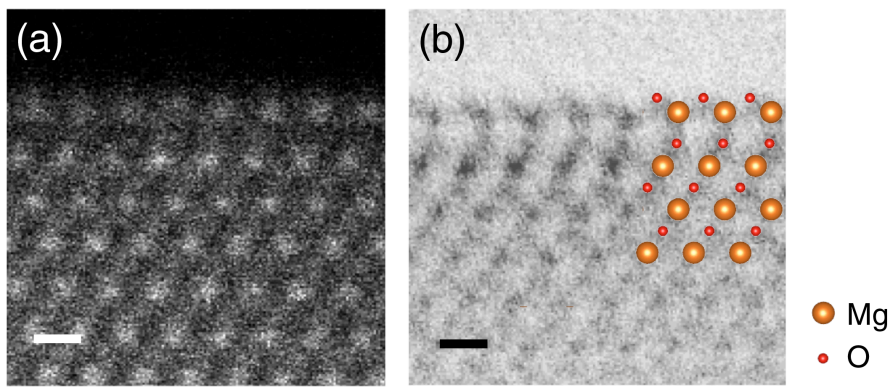

Figure 3. In-situ STEM observation of $\mathrm{MgO}$ (111) surface reconstruction viewed along $<110>$, (a) HAADF-STEM (b) ABF-STEM, scale bar $0.26 \mathrm{~nm}$. 